\title{
An appraisal of analytical tools used in predicting clinical outcomes following radiation therapy treatment of men with prostate cancer: a systematic review
}

Elspeth Raymond ${ }^{1}$, Michael E. O'Callaghan ${ }^{1,2,7,8^{*}}$, Jared Campbell ${ }^{9}$, Andrew D. Vincent ${ }^{1,2}$, Kerri Beckmann ${ }^{1,3}$, David Roder ${ }^{3}$, Sue Evans ${ }^{4}$, John McNeil ${ }^{4}$, Jeremy Millar ${ }^{5}$, John Zalcberg ${ }^{4,11}$, Martin Borg ${ }^{6}$ and Kim Moretti ${ }^{1,2,3,9,10}$

\begin{abstract}
Background: Prostate cancer can be treated with several different modalities, including radiation treatment. Various prognostic tools have been developed to aid decision making by providing estimates of the probability of different outcomes. Such tools have been demonstrated to have better prognostic accuracy than clinical judgment alone.

Methods: A systematic review was undertaken to identify papers relating to the prediction of clinical outcomes (biochemical failure, metastasis, survival) in patients with prostate cancer who received radiation treatment, with the particular aim of identifying whether published tools are adequately developed, validated, and provide accurate predictions. PubMed and EMBASE were searched from July 2007. Title and abstract screening, full text review, and critical appraisal were conducted by two reviewers. A review protocol was published in advance of commencing literature searches.

Results: The search strategy resulted in 165 potential articles, of which 72 were selected for full text review and 47 ultimately included. These papers described 66 models which were newly developed and 31 which were external validations of already published predictive tools. The included studies represented a total of 60,457 patients, recruited between 1984 and 2009. Sixty five percent of models were not externally validated, 57\% did not report accuracy and $31 \%$ included variables which are not readily accessible in existing datasets. Most models $(72,74 \%)$ related to external beam radiation therapy with the remainder relating to brachytherapy (alone or in combination with external beam radiation therapy).

Conclusions: A large number of prognostic models (97) have been described in the recent literature, representing a rapid increase since previous reviews (17 papers, 1966-2007). Most models described were not validated and a third utilised variables which are not readily accessible in existing data collections. Where validation had occurred, it was often limited to data taken from single institutes in the US. While validated and accurate models are available to predict prostate cancer specific mortality following external beam radiation therapy, there is a scarcity of such tools relating to brachytherapy. This review provides an accessible catalogue of predictive tools for current use and which should be prioritised for future validation.
\end{abstract}

Keywords: Prostate cancer, Systematic literature review, Nomogram, Outcomes, Survival, Biochemical recurrence

\footnotetext{
* Correspondence: Michael.ocallaghan@health.sa.gov.au

'South Australian Prostate Cancer Clinical Outcomes Collaborative

(SA-PCCOC), Adelaide, Australia

${ }^{2}$ Freemasons Foundation Centre for Men's Health, University of Adelaide,

Adelaide, Australia

Full list of author information is available at the end of the article
} 


\section{Background Rationale}

Prostate cancer is the most prevalent cancer in men globally, with 1.4 million new cases reported in 2013 [1]. Prostate cancer cases increased by $217 \%$ between 1990 and 2013 as a result of population growth and aging and increased uptake of opportunistic screening, particularly in developing countries [1]. Prostate cancer remains the leading cause of death among males in 24 of 188 countries covered by the Global Burden of Disease Cancer Collaboration [1].

Prostate cancer treatments are varied and include: deferred treatment (active surveillance), watchful waiting, radical prostatectomy, radiation therapy (with or without androgen deprivation therapy) or androgen deprivation therapy (ADT) [2, 3]. Each treatment will achieve different outcomes in terms of oncology (e.g., survival or time to biochemical recurrence), adverse events and patient reported outcomes such as urinary incontinence and impotence. These outcomes are important considerations when selecting a treatment for prostate cancer patients and are considered in the context of patient age, life expectancy, co-morbidities, tumour size, grade and stage and other risk indicators that influence outcomes and treatment choice. Determining which treatment choice is optimal for each patient remains an important challenge, particularly where directly relevant randomised controlled data is lacking.

To aid this decision making process, a number of tools have been developed with nomograms and risk stratification systems most commonly used [4]. Nomograms are graphic tools developed to aid clinical decision making and are well established in clinical practice for prostate cancer, particularly for assisting selection of treatment approaches based on risk stratification. Such tools have been shown to improve prediction of outcomes when compared with clinician judgement alone $[5,6]$. Unfortunately most nomograms currently in use are likely to be based on dated treatment modalities. Furthermore predictions based on observations made in one setting may not be accurate in another (e.g., where ethnicity or health services differ). Extrapolation of published international results to local practice is a known pitfall that has potential to mislead both clinicians and patients [7]. These limitations are particularly relevant to predictive tools designed for use in patients treated with radiation therapy as this modality has changed significantly over the past decade.

\section{Objectives}

We aim to identify papers predicting clinical outcomes for patients with prostate cancer who have been treated with radiation therapy. We particularly set out to assess if the tools identified were adequately developed, validated and provide accurate predictions.

\section{Methods \\ Protocol and registration}

A systematic literature review protocol was developed for this study and registered before searches commenced with PROSPERO, an international prospective register of systematic reviews. The protocol can be accessed at: http://www.crd.york.ac.uk/PROSPERO/display_record.asp? ID=CRD42015025428.

\section{Inclusion criteria}

Papers were eligible for inclusion where they met the following criteria; Population: Patients with prostate cancer. Exposure: Treatment with radiation therapy (including external beam radiation therapy and/or brachytherapy). Outcome: The generation or validation of a tool for the prediction of clinical outcomes (biochemical failure $[\mathrm{BF}]$, progression to metastases, prostate cancer specific survival, overall survival). Papers had to be written in English and published post July 2007. This date was chosen as it is the search date up to which a previous systematic review of prognostic tools for prostate cancer treated by any therapy was undertaken [4]. Studies were included which described tools using variables which are currently available in a clinical setting. This excluded papers including genetic or molecular variables.

\section{Information sources}

Searches were conducted of the Medline database (PubMed interface) and the EMBASE database.

\section{Search}

Disease-specific search terms included: prostate cancer, prostatic neoplasms, cancer of the prostate, adenocarcinoma of the prostate, prostatic cancer, prostate gland cancer and prostate tumour. Treatment specific search terms included: radiation therapy, radiotherapy, external beam radiotherapy, EBRT, brachytherapy, high dose radiotherapy, low dose radiotherapy and targeted radiotherapies. Outcome-specific search terms included: overall survival, progression-free survival, PFS, mortality, event free survival, EFS, disease free survival, prostate cancer specific survival, progression to metastases, time to progression, TTP, biochemical recurrence, BCR, biochemical failure, neoplasm recurrence. Search terms used to identify predictive models included: predictive tools, nomograms, risk stratification, Partin tables, regression tree analysis, Artificial Neural Networks, CAPRA-S or CAPRA score, risk estimates, algorithms, predictive accuracy, diagnostic test accuracy, Kattan tables/ nomograms. 


\section{Study selection}

Study selection included three phases. The titles and abstracts of all studies identified by the search strategy were compared to the inclusion criteria detailed above by two authors working independently (ER and MOC). All studies that appeared likely to meet the inclusion criteria were progressed to full-text review. All discrepancies, where authors reached different conclusions about the same papers, were resolved through discussion. The full-texts of these papers were then retrieved and assessed against the inclusion criteria, again by two authors (ER, JC or MOC) working independently in order to minimise the impact of human error. Studies that were identified as meeting all inclusion criteria were included in the review, while those which did not were excluded. Again, where there were differences in the authors' conclusions consensus on the correct decision was reached through discussion. Finally, the reference lists of included papers were screened for any additional relevant papers which may have been missed by the search strategy. All new titles identified were then reviewed as described above.

\section{Data collection process and data items}

After full text review, data extraction was undertaken by one reviewer (ER, JC or MOC). Items for extraction included: manuscript identifiers (author, contact, country, setting), study methods, population studied (inclusion criteria, exclusion criteria, baseline characteristics dates of recruitment, age, ethnicity, number of patients, primary treatment, treatment subtype, adjuvant therapies, neoadjuvant therapies), and predictive model characteristics (type of model, variables included, if internal validation was reported and the type, external validation, variable definitions, if variables were readily available, sample size, number of events, definition of outcome, model accuracy, sensitivity, specificity, concordance index and receiver operator curve area under the curve). For assessment as to whether or not variables were considered 'readily available' the minimum data set used by the only national prostate cancer registry (Prostate Cancer Outcomes Registry, Australia and New Zealand Australian [8]) was used as a guide.

\section{Quality assessment}

Quality assessment was performed by two reviewers (ER, JC or MOC) for each paper. Four questions were selected for this assessment: 1 . Was the defined representative sample of patients assembled at a common (usually early) point in the course of their disease? 2. Was patient follow-up sufficiently long and complete? 3 . Were outcome criteria either objective or applied in a 'blind' fashion? And 4. If subgroups with different prognoses were identified, did adjustment for important prognostic factors take place? These questions were selected from the Centre for Evidence Based Medicine 'Critical appraisal of prognostic studies' tool [9]. Discrepancies between reviewers were discussed and consensus reached. Questions that were answered positively $>75 \%$ of the time were considered to present a low risk of bias, those $\leq 75$ to $>50 \%$ a moderate risk of bias, and any $\leq 50 \%$ a high risk of bias. Data extraction and quality assessment were performed using the online tool 'Covidence'.

\section{Results}

The search strategy resulted in 165 potentially relevant abstracts/articles and these were reduced to 72 once duplicates were removed and title and abstracts were screened (Fig. 1). The full-text of these papers was reviewed against the inclusion criteria (reasons for exclusion are reported in Additional file 1: Table S1a and b) and 47 finally selected. Study recruitment periods varied considerably with the earliest patients being from 1984 [10] and the latest 2009 [10-13] (Table 1). The populations of individual studies varied from 80 [14] to 7,839 $[14,15]$ with a combined population of 60,457 (Tables 2 , 3 and 4 ). The majority of studies were retrospective $(n=38)$, however seven studies recruited prospective cohorts (for one study [16] it was not stated whether it was retrospective or prospective).

The 47 papers finally included in this review described 97 individual predictive models. Of these models, 16 related to brachytherapy treatment (Table 2), 72 to external beam radiation therapy (Table 3 ) and nine to a combination of brachytherapy and external beam radiation therapy (Table 4).

Across all radiation treatment modalities, outcomes relating to PSA levels post treatment were most common (39 models) followed by prostate cancer specific mortality (29 models). Measures of metastases (17) and overall survival (14 models) were less common (note that some papers report more than one outcome and model). Of those studies reporting development of new

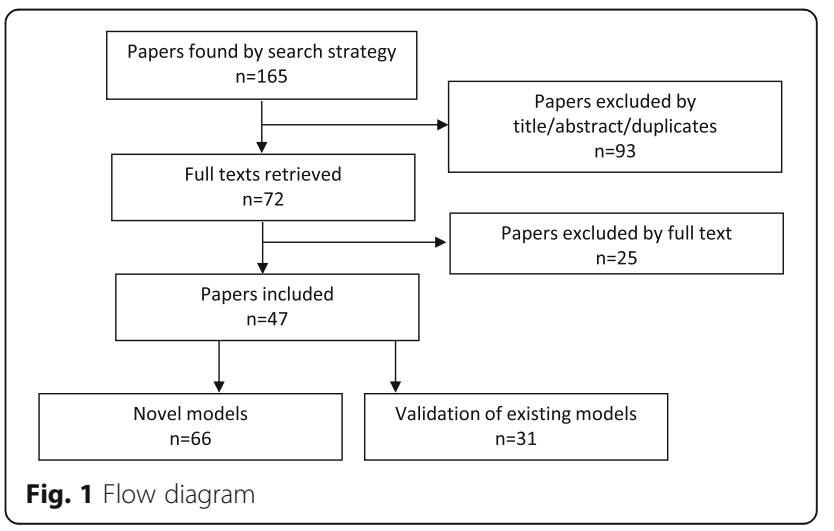


Table 1 Summary of papers describing prognostic tools relating to clinical outcomes following radiation therapy (2007-2015)

\begin{tabular}{|c|c|c|c|c|c|c|}
\hline Author & $\begin{array}{l}\text { Recruitment } \\
\text { window }\end{array}$ & Country & Population & Outcome & Study type & Setting \\
\hline Bittner [27] & 1995-2006 & USA & $\begin{array}{l}\text { Prostate cancer patients } \\
\text { treated with brachytherapy }\end{array}$ & BFFF, PCSM & Retrospective & Single centre \\
\hline $\begin{array}{l}\text { Buyyounouski } \\
\text { [38] }\end{array}$ & 1989-2000 & $\begin{array}{l}\text { Canada, } \\
\text { Aust, USA }\end{array}$ & $\begin{array}{l}\text { Men previously treated with } \\
\text { EBRT for clinically localized } \\
\text { prostate adenocarcinoma } \\
\text { and subsequently diagnosed } \\
\text { with BCF. }\end{array}$ & PCSM & Retrospective & Multi-centre \\
\hline $\begin{array}{l}\text { Cooperberg } \\
\text { [39] }\end{array}$ & 1995-2007 & USA & Men enrolled in CaPSURE & PCSM & Retrospective & $\begin{array}{l}\text { Multi-centre } \\
\text { (CaPSURE } \\
\text { Registry) }\end{array}$ \\
\hline $\begin{array}{l}\text { Cooperberg } \\
{[40]}\end{array}$ & 1995-2008 & USA & $\begin{array}{l}\text { Men with localized disease } \\
\text { who underwent prostatectomy, } \\
\text { received external-beam } \\
\text { radiation, or received primary } \\
\text { androgen deprivation; and } \\
\text { had at least } 6 \text { months of } \\
\text { follow-up recorded. }\end{array}$ & 10 year PCSM & Retrospective & $\begin{array}{l}\text { Multi-centre } \\
\text { (CaPSURE } \\
\text { Registry) }\end{array}$ \\
\hline $\begin{array}{l}\text { D'Ambrosio } \\
{[41]}\end{array}$ & 1989-2004 & USA & $\begin{array}{l}\text { Men with prostate cancer } \\
\text { treated with RT. }\end{array}$ & BCF & Retrospective & Single centre \\
\hline D'Amico [42] & $1991-2005$ & USA & $\begin{array}{l}\text { Men with high-risk prostate } \\
\text { cancer (locally or advanced) } \\
\text { and } 10 \text { year life expectancy } \\
\text { treated with brachytherapy } \\
\text { who were observed for a min } \\
\text { of } 2 \text { years. }\end{array}$ & $\begin{array}{l}\text { PCSM and presence } \\
\text { of hormone-refractory } \\
\text { metastatic prostate } \\
\text { cancer. }\end{array}$ & Prospective & Multi-centre \\
\hline D'Amico [43] & 1988-2004 & USA & $\begin{array}{l}\text { Men who underwent RT for } \\
\text { prostate cancer for at least } \\
1 \text { high-risk feature. }\end{array}$ & PCSM & Prospective & Multi-centre \\
\hline Delouya [19] & $\begin{array}{l}\text { 2002-Not } \\
\text { stated }\end{array}$ & Canada & $\begin{array}{l}\text { Men with low or intermediate-risk } \\
\text { prostate cancer treated with } \\
\text { brachytherapy, EBRT within a } \\
\text { phase II or III research protocol, } \\
\text { or ERBT outside of a protocol. }\end{array}$ & $\mathrm{BCF}$ & Retrospective & Single centre \\
\hline Denham [44] & 1996-2000 & $\begin{array}{l}\text { Australia \& } \\
\text { New Zealand }\end{array}$ & $\begin{array}{l}\text { Men with locally advanced } \\
\text { prostate cancer receiving RT }\end{array}$ & PCSM & Prospective & Multi-centre \\
\hline Engineer [9] & 1984-2004 & India & $\begin{array}{l}\text { Patients with a histological } \\
\text { diagnosis of prostate cancer }\end{array}$ & $\begin{array}{l}\text { BFFF, PCSM, DM, } \\
\text { BCF, OS }\end{array}$ & Retrospective & Single centre \\
\hline Feng [28] & 1998-2008 & USA & $\begin{array}{l}\text { Men with clinically localized } \\
\text { prostate cancer treated with } \\
\text { EBRT. }\end{array}$ & $\begin{array}{l}\text { FFM, PCSM, BFFF, } \\
\text { OS }\end{array}$ & Retrospective & Single centre \\
\hline Frank [45] & 1996-2006 & $\begin{array}{l}\text { USA, Canada, } \\
\text { Netherlands. }\end{array}$ & $\begin{array}{l}\text { Men with prostate cancer } \\
\text { treated with brachytherapy } \\
\text { with at least } 30 \text { months of } \\
\text { follow-up. }\end{array}$ & PSA failure. & Retrospective & Multi -centre \\
\hline Frank [25] & 1998-2006 & USA & $\begin{array}{l}\text { Men with prostate cancer } \\
\text { treated with permament } \\
125 \text { I brachytherapy. }\end{array}$ & 5 year BFFF & Retrospective & Single centre \\
\hline Halverson [46] & 1998-2008 & USA & $\begin{array}{l}\text { Men with clinically localized } \\
\text { prostate cancer treated with } \\
\text { EBRT with or without adjuvant } \\
\text { ADT }\end{array}$ & BFFF & Retrospective & Single centre \\
\hline Huang [47] & 1993-2003 & USA, Australia & $\begin{array}{l}\text { Men with clinical Stage } \\
\text { T1c-T3N0M0 prostate } \\
\text { adenocarcinoma treated with } \\
\text { EBRT with or without a high-dose } \\
\text { rate brachytherapy boost. }\end{array}$ & $\begin{array}{l}\text { BCF, DM, PCSM, } \\
\text { OS. }\end{array}$ & Retrospective & Single centre \\
\hline Kaplan [12] & 2000-2009 & Israel & Patients with prostate cancer & BFFF & Retrospective & Single centre \\
\hline
\end{tabular}


Table 1 Summary of papers describing prognostic tools relating to clinical outcomes following radiation therapy (2007-2015) (Continued)

\begin{tabular}{|c|c|c|c|c|c|c|}
\hline Krishnan [20] & $2003-2008$ & Canada & $\begin{array}{l}\text { Men with intermediate-risk } \\
\text { prostate cancer with } \\
\text { a minimum follow-up of } \\
3 \text { years. }\end{array}$ & $\mathrm{BCF}$ & Retrospective & Single centre \\
\hline Kubicek [48] & 1998-2004 & USA & $\begin{array}{l}\text { Men with biopsy proven } \\
\text { T1-T2 prostate adenocarcinoma } \\
\text { treated with EBRT \& LDR. }\end{array}$ & CSS & Retrospective & Single centre \\
\hline Marshall [11] & 1990-2009 & USA & $\begin{array}{l}\text { Men treated with brachytherapy } \\
\text { for biopsy-proven prostate } \\
\text { adenocarcinoma. }\end{array}$ & $B C F$ & Retrospective & Single centre \\
\hline McKenna [49] & 1998-2003 & USA & $\begin{array}{l}\text { Men with biopsy-proved } \\
\text { prostate cancer who had } \\
\text { MRI imaging prior to EBRT. }\end{array}$ & $\begin{array}{l}\text { Metastatic } \\
\text { recurrence } \\
\text { and } B C F\end{array}$ & Retrospective & Single centre \\
\hline Murgic [50] & 1998-2008 & USA & $\begin{array}{l}\text { Men with clinically localized } \\
\text { prostate adenocarcinoma } \\
\text { treated with EBRT. }\end{array}$ & $\begin{array}{l}\text { BFFF, FFM, } \\
\text { PCSM and OS }\end{array}$ & Retrospective & Single centre \\
\hline Potters [16] & Not stated & USA & $\begin{array}{l}\text { Prostate cancer patients } \\
\text { treated with brachytherapy. }\end{array}$ & 9-year BFFF & Retrospective & Multi-centre \\
\hline $\begin{array}{l}\text { Proust-Lima } \\
{[51]}\end{array}$ & Not stated & USA & $\begin{array}{l}\text { Men treated for localized } \\
\text { prostate cancer with EBRT. }\end{array}$ & $\mathrm{BCF}$ & Prospective & Multi-centre \\
\hline Qian [52] & 1998-2008 & USA & $\begin{array}{l}\text { Men who were treated with } \\
\text { EBRT for clinically localized } \\
\text { prostate cancer with or } \\
\text { without neoadjuvant or } \\
\text { adjuvant ADT. }\end{array}$ & $\begin{array}{l}\text { BFFF, FFM, } \\
\text { OS, PCSM. }\end{array}$ & Retrospective & Single centre \\
\hline Rodrigues [14] & Not stated & Canada & Men with prostate cancer. & BFFF, OS & Retrospective & $\begin{array}{l}\text { Multi-centre } \\
\text { (GUROC } \\
\text { ProCaRS } \\
\text { database) }\end{array}$ \\
\hline Sabolch [53] & 1998-2008 & USA & $\begin{array}{l}\text { Men treated for localized } \\
\text { prostate cancer with EBRT. }\end{array}$ & $\begin{array}{l}\text { BFFF, FFM, } \\
\text { OS, PCSM. }\end{array}$ & Prospective & Single centre \\
\hline Sanpaolo [21] & $2000-2004$ & Italy & $\begin{array}{l}\text { Men with T1-T3 NO prostate } \\
\text { cancer. }\end{array}$ & BCF & Retrospective & Single centre \\
\hline Slater [54] & 1991-1999 & USA & $\begin{array}{l}\text { Randomly selected prostate } \\
\text { cancer patients treated with } \\
\text { proton and photon beam } \\
\text { therapy. }\end{array}$ & bNED & Retrospective & Single centre \\
\hline Spratt [55] & 1997-2008 & USA & $\begin{array}{l}\text { Men with localized prostate } \\
\text { cancer were treated with IMRT. }\end{array}$ & $\begin{array}{l}\text { BCF, DMFS, } \\
\text { BCR }\end{array}$ & Retrospective & Single centre \\
\hline Steigler [56] & $1996-2000$ & $\begin{array}{l}\text { Australia \& } \\
\text { New Zealand }\end{array}$ & $\begin{array}{l}\text { Men with localised advanced } \\
\text { prostate cancer treated with RT } \\
\text { and experienced BCF prior to } \\
\text { clinical failure or secondary } \\
\text { theraputic intervention. }\end{array}$ & $\begin{array}{l}\text { TTBF, PCSM, } \\
\text { distant } \\
\text { progression } \\
\text { and STI from BCF }\end{array}$ & Retrospective & Multi-centre \\
\hline Sylvester [57] & 1988-1992 & USA & $\begin{array}{l}\text { Men with clinically localized } \\
\text { prostate cancer treated with } \\
\text { implanted I-125. }\end{array}$ & $\begin{array}{l}15 \text { year BFFF, } \\
\text { CSS and OS. }\end{array}$ & Prospective & $\begin{array}{l}\text { Consecutive } \\
\text { case series }\end{array}$ \\
\hline Taylor [58] & Not stated & USA & $\begin{array}{l}\text { Men with localized prostate } \\
\text { cancer,NO/MO treated with RT. }\end{array}$ & $\begin{array}{l}\text { Clinical recurrence } \\
\text { (local, regional or distant) }\end{array}$ & Retrospective & Multi-centre \\
\hline Thames [59] & 1987-1995 & USA & $\begin{array}{l}\text { Men with clinical stages } \mathrm{T} 1 \mathrm{~b}, \\
\mathrm{~T} 1 \mathrm{c} \text {, and } \mathrm{T} 2 \mathrm{~N} 0 \mathrm{M0} \text { biopsy } \\
\text { proven prostate adenocarcinoma. }\end{array}$ & $\mathrm{BCF}$ & Retrospective & Multi-centre \\
\hline $\begin{array}{l}\text { Vainshtein } \\
{[18]}\end{array}$ & 1998-2008 & USA & $\begin{array}{l}\text { Men with localized prostate } \\
\text { cancer treated with EBRT, } \\
+/- \text { ADT }\end{array}$ & FFM, PCSM. & Prospective & Single centre \\
\hline
\end{tabular}


Table 1 Summary of papers describing prognostic tools relating to clinical outcomes following radiation therapy (2007-2015) (Continued)

\begin{tabular}{|c|c|c|c|c|c|c|}
\hline Vance $[60]$ & $1998-2008$ & USA & $\begin{array}{l}\text { Men with clinically localized } \\
\text { prostate cancer treated with } \\
\text { EBRT, with or without neoadjuvant } \\
\text { or adjuvant ADT. }\end{array}$ & BFFF, DMFS, PCSM \& OS. & Retrospective & Single centre \\
\hline Wattson [61] & 1991-2007 & USA & $\begin{array}{l}\text { Men with high-risk prostate } \\
\text { cancer. }\end{array}$ & PCSM & Retrospective & Multicentre \\
\hline $\begin{array}{l}\text { Westphalen } \\
{[62]}\end{array}$ & 1998-2007 & USA & $\begin{array}{l}\text { Prostate cancer patients who } \\
\text { underwent endorectal } \\
\text { MR and MR spectroscopy } \\
\text { prior to EBRT. }\end{array}$ & $B C F$ & Retrospective & $\begin{array}{l}\text { Multi-centre } \\
\text { (national } \\
\text { administrative } \\
\text { data set) }\end{array}$ \\
\hline Williams [17] & 1991-2002 & $\begin{array}{l}\text { US, Canada, } \\
\text { Australia }\end{array}$ & $\begin{array}{l}\text { Men with clinical T1-4 N0/X M0/X } \\
\text { prostate adenocarcinoma treated } \\
\text { with EBRT. }\end{array}$ & $\mathrm{BCF}$ & Retrospective & Multi-centre \\
\hline Yoshida [15] & $2003-2008$ & Japan & $\begin{array}{l}\text { Men with histologically-proven } \\
\text { prostate adenocarcinoma, treated } \\
\text { with HDR-ISBT. }\end{array}$ & $\begin{array}{l}5 \text { year PSA } \\
\text { failure and OS }\end{array}$ & Retrospective & Single centre \\
\hline Yu [63] & 1987-2001 & USA & $\begin{array}{l}\text { Men with prostate cancer treated } \\
\text { with EBRT. }\end{array}$ & $B C F$ & Retrospective & Single centre \\
\hline Yu [64] & 1993-2002 & USA & $\begin{array}{l}\text { Men newly diagnosed with } \\
\text { clinically node-negative, localized } \\
\text { adenocarcinoma of the prostate } \\
\text { treated with EBRT. }\end{array}$ & $\mathrm{BCF}$ & Retrospective & Single centre \\
\hline Zaorsky [65] & 1992-2004 & USA & $\begin{array}{l}\text { Men with clinical stage T1-4, } \\
\text { NO/NX-N1, MO adenocarcinoma } \\
\text { of the prostate received RT with } \\
\text { or without adjuvant ADT. }\end{array}$ & $\mathrm{BCF}, \mathrm{DM}, \mathrm{OS}$ & Retrospective & Single centre \\
\hline Zelefsky [66] & 1988-2004 & USA & $\begin{array}{l}\text { Men with clinically staged T1-T3 } \\
\text { node-negative prostate cancer } \\
\text { treated with 3D-CRT or IMRT. }\end{array}$ & DMFS, BFFF. & Retrospective & Single centre \\
\hline Zelefsky [67] & $1998-2000$ & USA & $\begin{array}{l}\text { Men with clinically localized } \\
\text { prostate cancer treated with } \\
\text { 3D-CRT or IMRT. }\end{array}$ & DM,PCSM,BFFF & Retrospective & Single centre \\
\hline Zelefsky [68] & 1988-2004 & USA & $\begin{array}{l}\text { Men with Stage T1-T3 prostate } \\
\text { cancer treated with 3D-CRT } \\
\text { or IMRT. }\end{array}$ & PSA relapse & Retrospective & Single centre \\
\hline Zelefsky [10] & 1998-2009 & USA & $\begin{array}{l}\text { Men with clinically localised } \\
\text { prostate cancer treated with } \\
\text { brachytherapy. }\end{array}$ & BFFF & Retrospective & Single centre \\
\hline Zumsteg [69] & 1992-2007 & USA & $\begin{array}{l}\text { Men with intermediate-risk prostate } \\
\text { cancer, but without high-risk } \\
\text { features treated with EBRT. }\end{array}$ & BCF, BFFF, LF,PCSM, DM. & Retrospective & Single centre \\
\hline
\end{tabular}

Abbreviations: OS overall survival, CaPSURE Cancer of the Prostate Strategic Urologic Research Endeavour, $R T$ radiotherapy, $B C F$ bio chemical failure, $B F F F$ bio chemical freedom from failure, $P C S M$ prostate cancer specific mortality, $P S A-R F S$ prostate-specific antigen recurrence-free survival, $L F$ local failure, $D M$ distant metastases, DMFS distant metastases-free survival, FFM freedom from metastases, HDR-ISBT high-dose-rate interstitial brachytherapy, $T$ TBF time to bio chemical failure, STI secondary therapeutic intervention, $b N E D$ bio chemical no evidence of diseaese, 2D-CRT 2D - Conformal radiotherapy, 3D-CRT 3D -Conformal radiotherapy, EBRT external beam radiotherapy, LDR brachytherapy low dose rate brachytherapy, NO/NX no nodal involvement, $I-125$ lodine 125 brachytherapy

models (66), only nine reported validation either internally or in an additional cohort. Only 67/97 (69\%) models included variables which were considered to be readily available in existing data sets.

Critical appraisal considered the criteria set by the CEBM appraisal tool for prognostic studies [9]. Risk of bias ranged from moderate $(\mathrm{Q} 1$; Was the defined representative sample of patients assembled at a common point in the course of their disease? (72\%), Q2; Was patient follow-up sufficiently long and complete? (64\%)) to low (Q3; Were outcome criteria either objective or applied in a 'blind' fashion? (85\%), Q4; If subgroups with different prognoses are identified, did adjustment for important prognostic factors take place? (91\%)) (Table 5).

\section{Brachytherapy}

In regards to models predicting outcomes following brachytherapy, Potters et al. [17] report the highest cindex in a model developed and internally validated using a cohort of 5,931 patients. This model predicts 9 year freedom from biochemical failure and remains to be validated externally. Eleven models relating to 


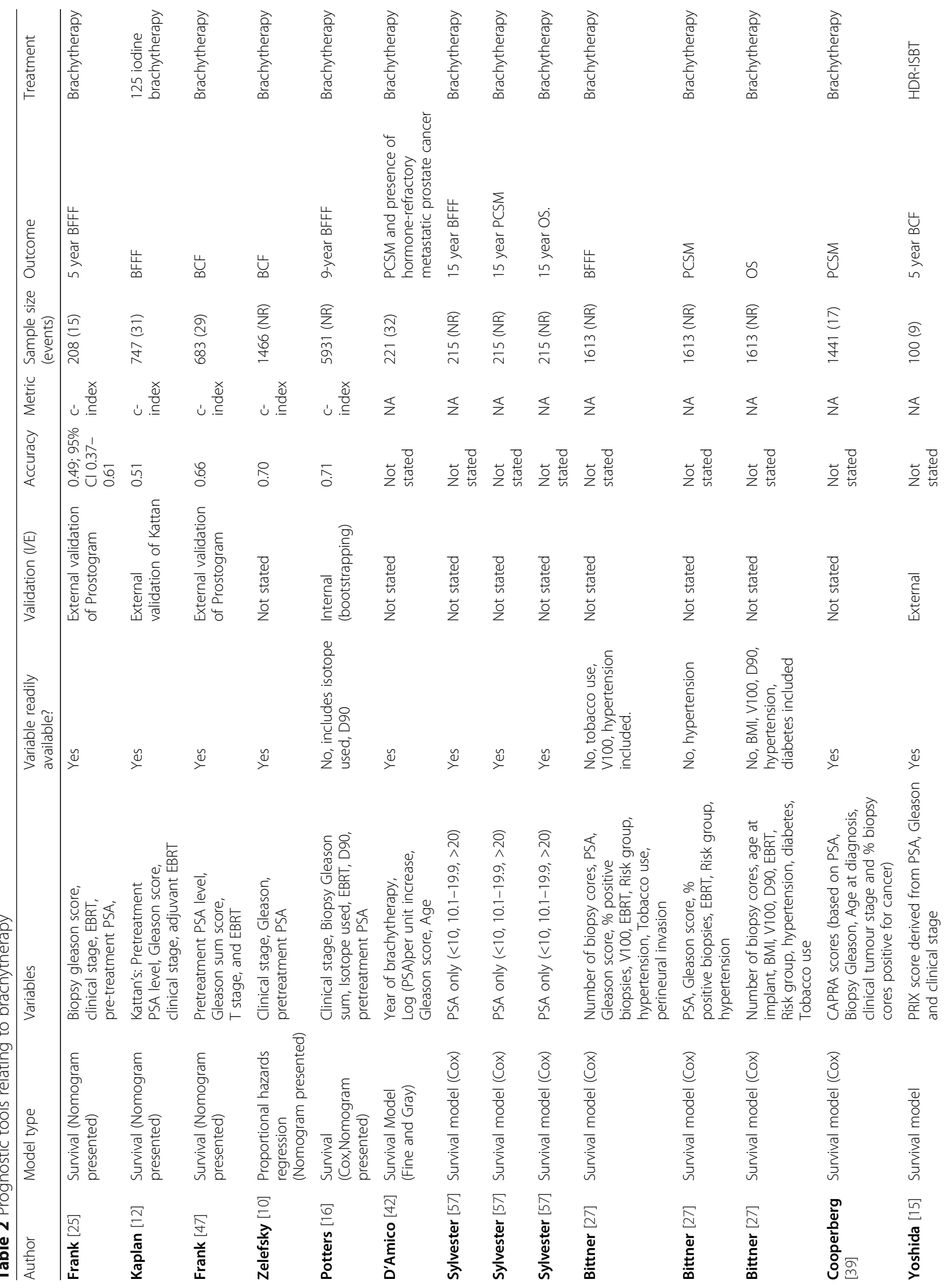




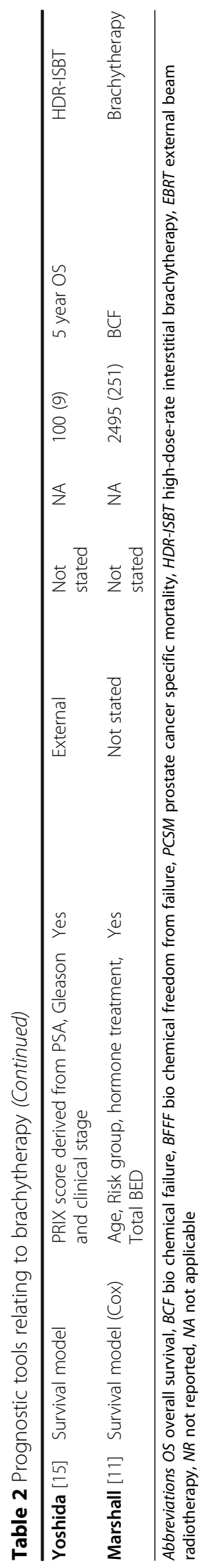




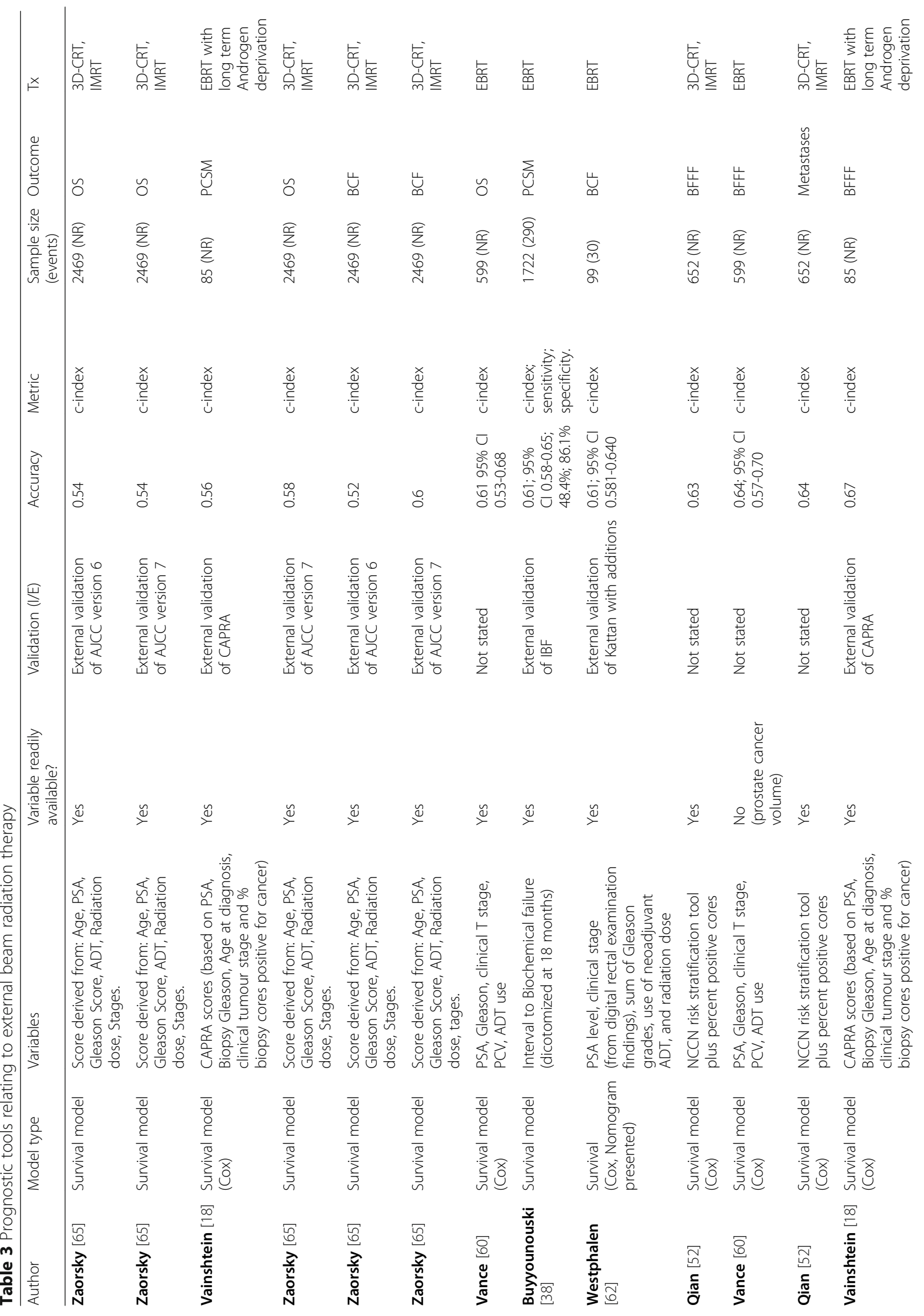




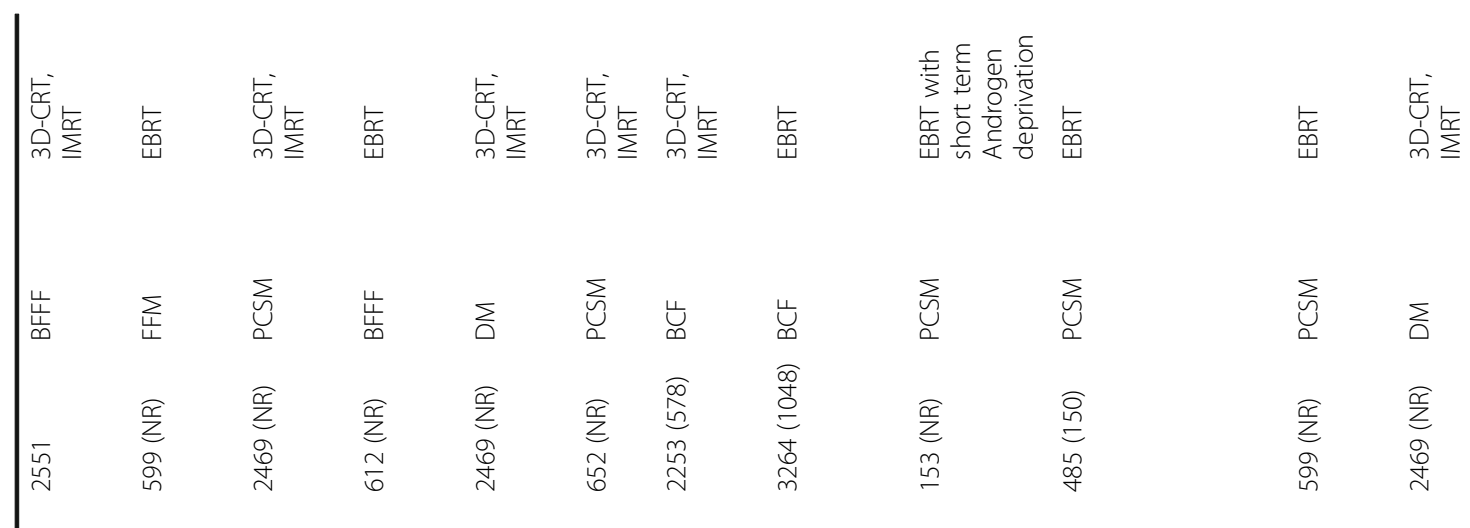

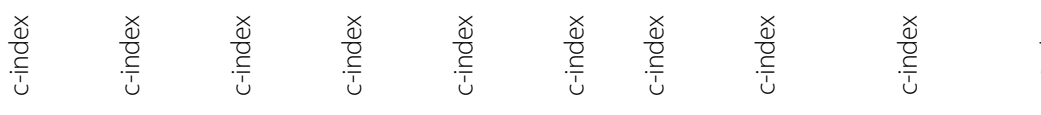

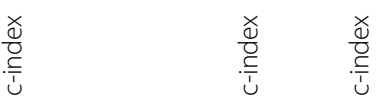

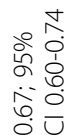

$\begin{array}{lll}0 & 0 \\ 0 & 0 & 0 \\ 0 & 0\end{array}$

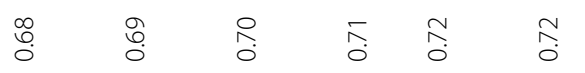

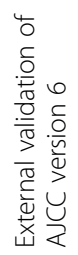

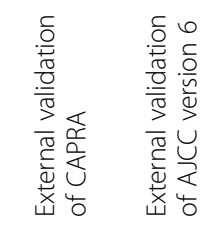

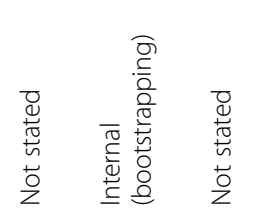

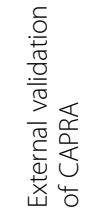

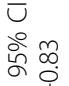

芒道:

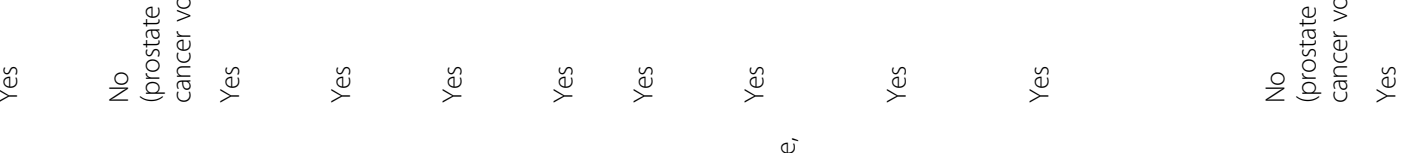

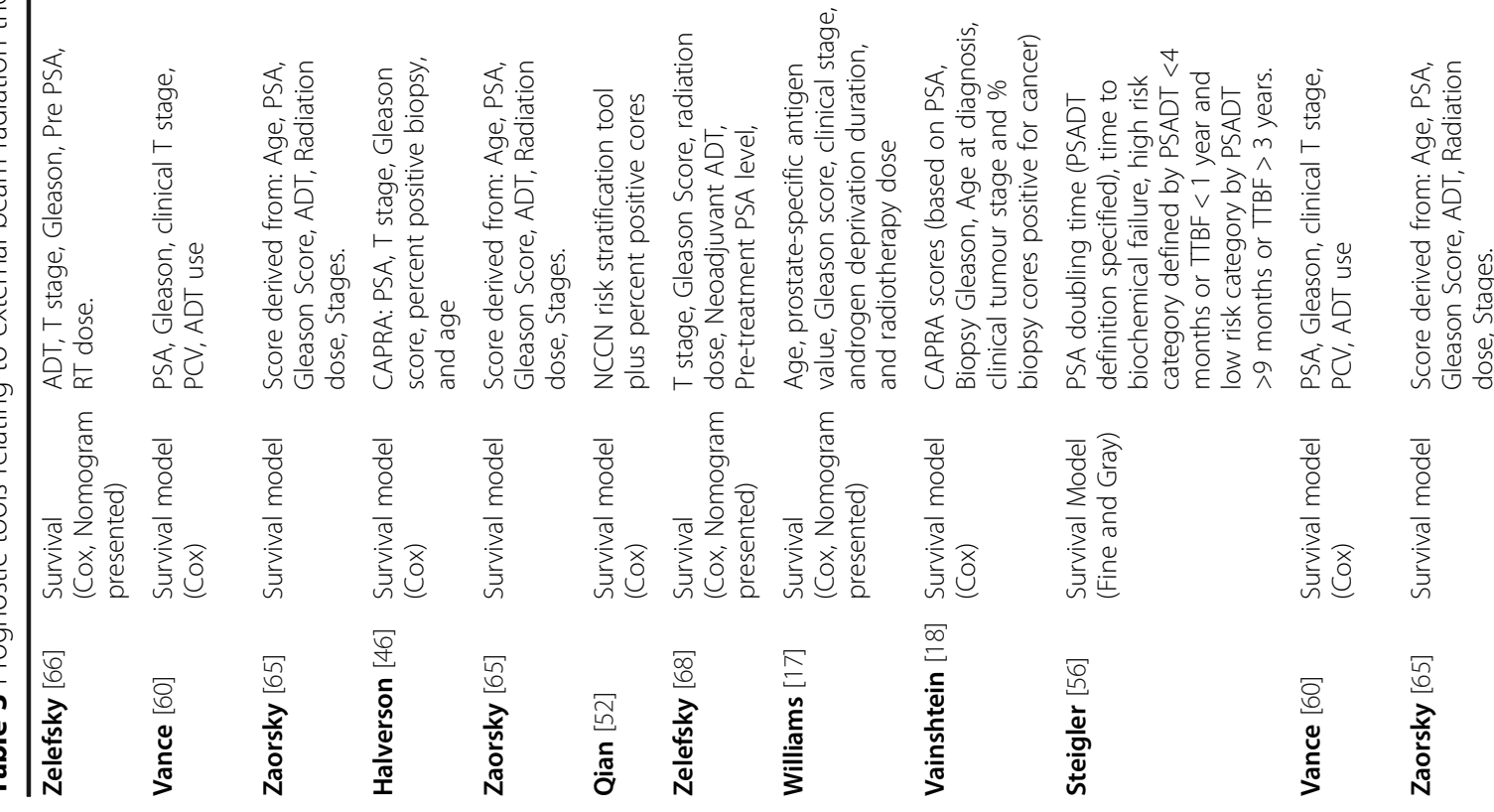




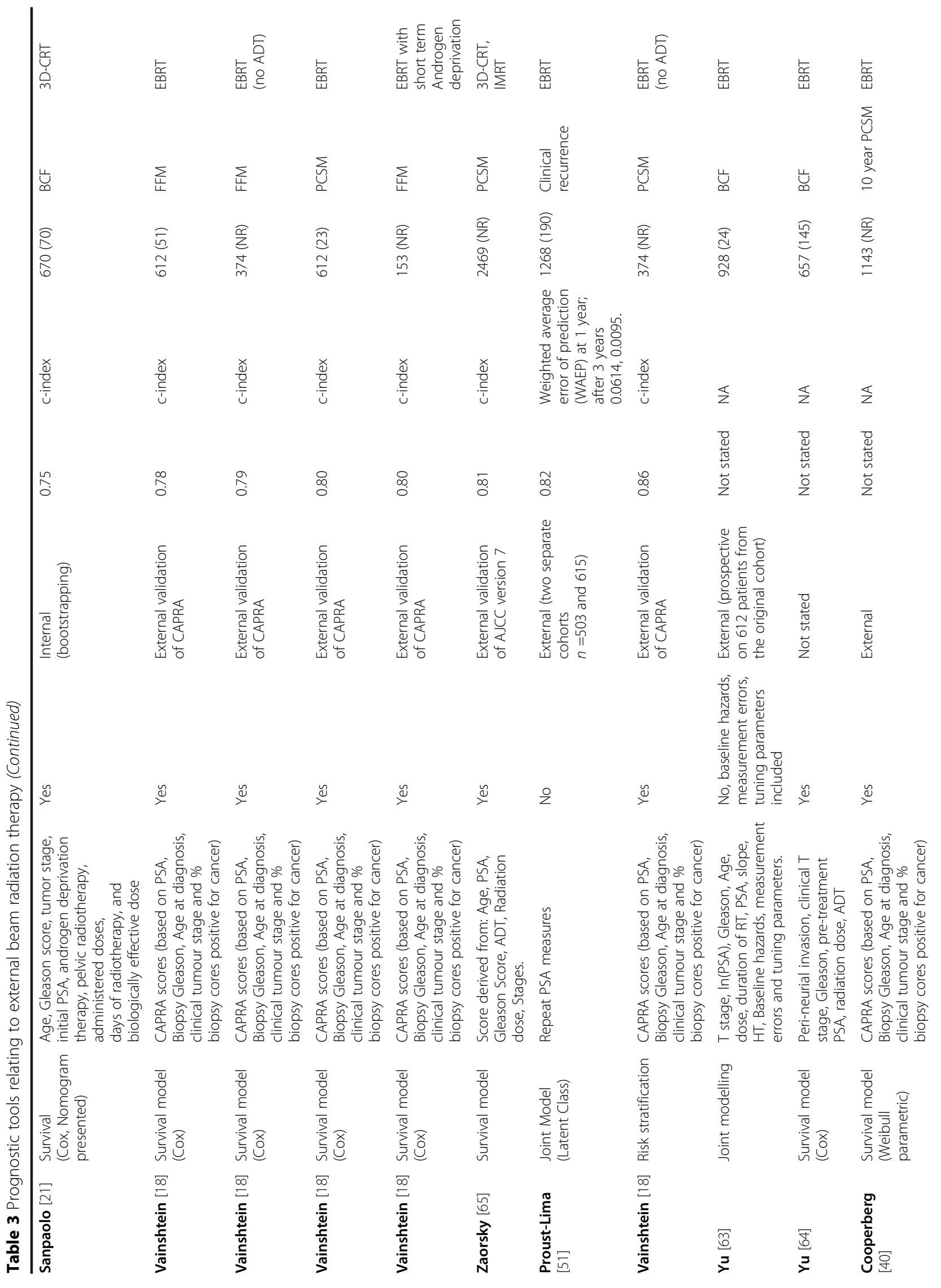




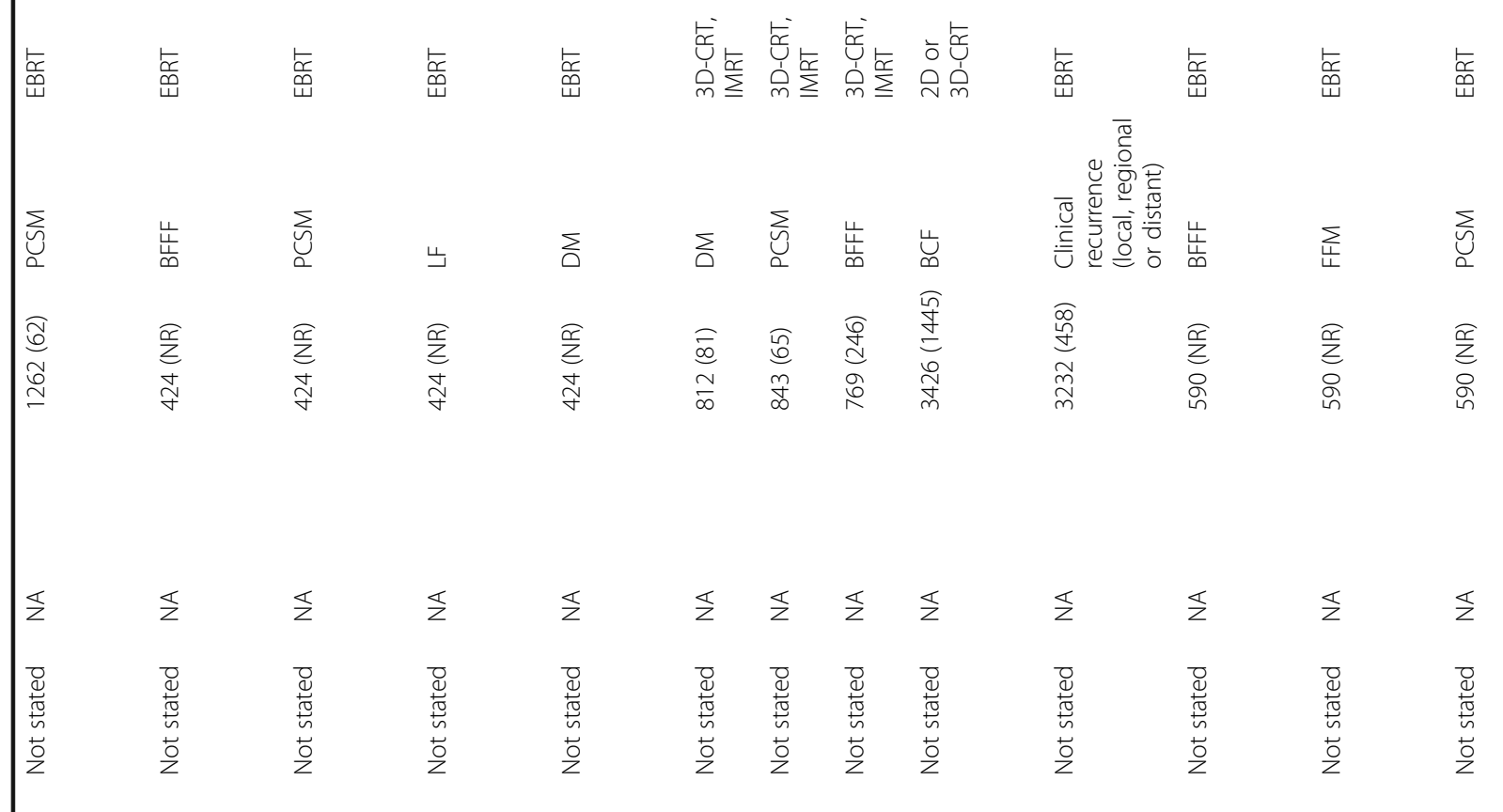

\begin{tabular}{|c|c|c|c|c|c|c|c|c|c|c|}
\hline 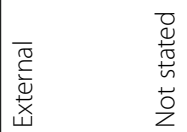 & $\begin{array}{l}\text { o } \\
\text { 节 } \\
\stackrel{0}{0}\end{array}$ & $\begin{array}{l}\text { o } \\
\text { 离 } \\
\text { to } \\
\text { t) }\end{array}$ & 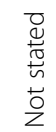 & 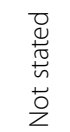 & 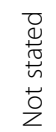 & 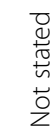 & 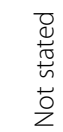 & 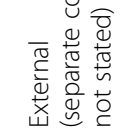 & & 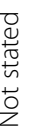 \\
\hline
\end{tabular}




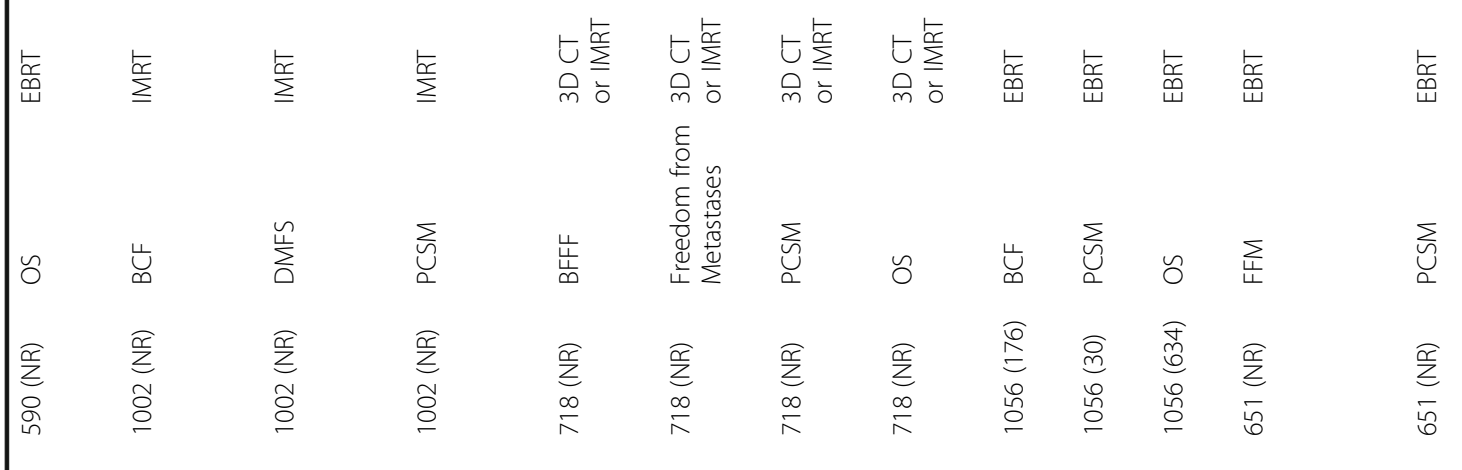

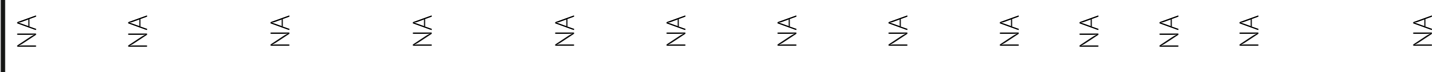

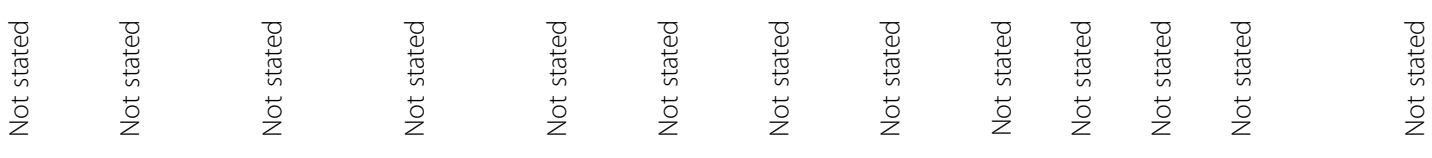

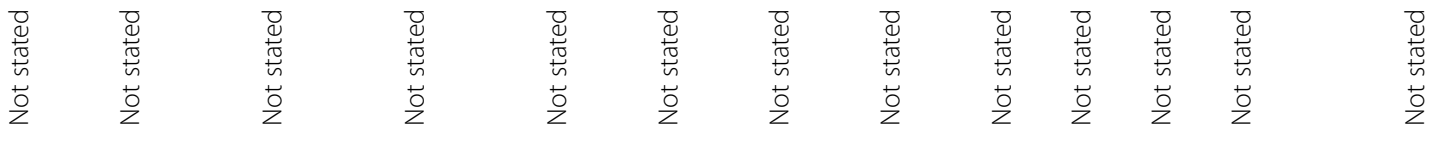

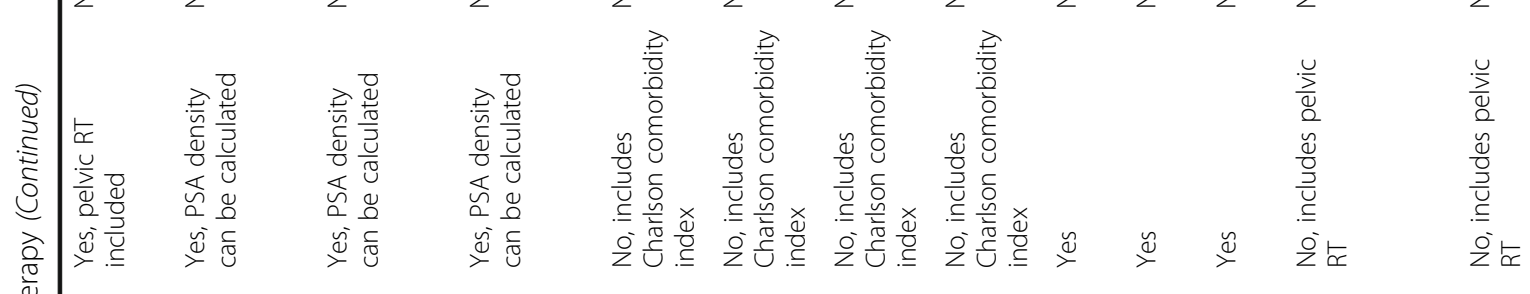

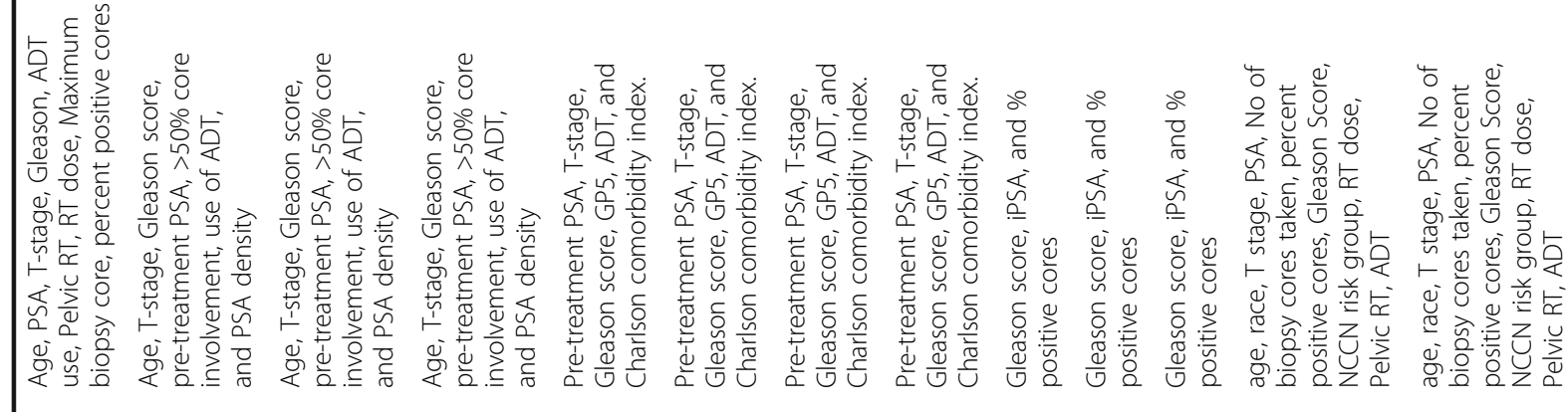




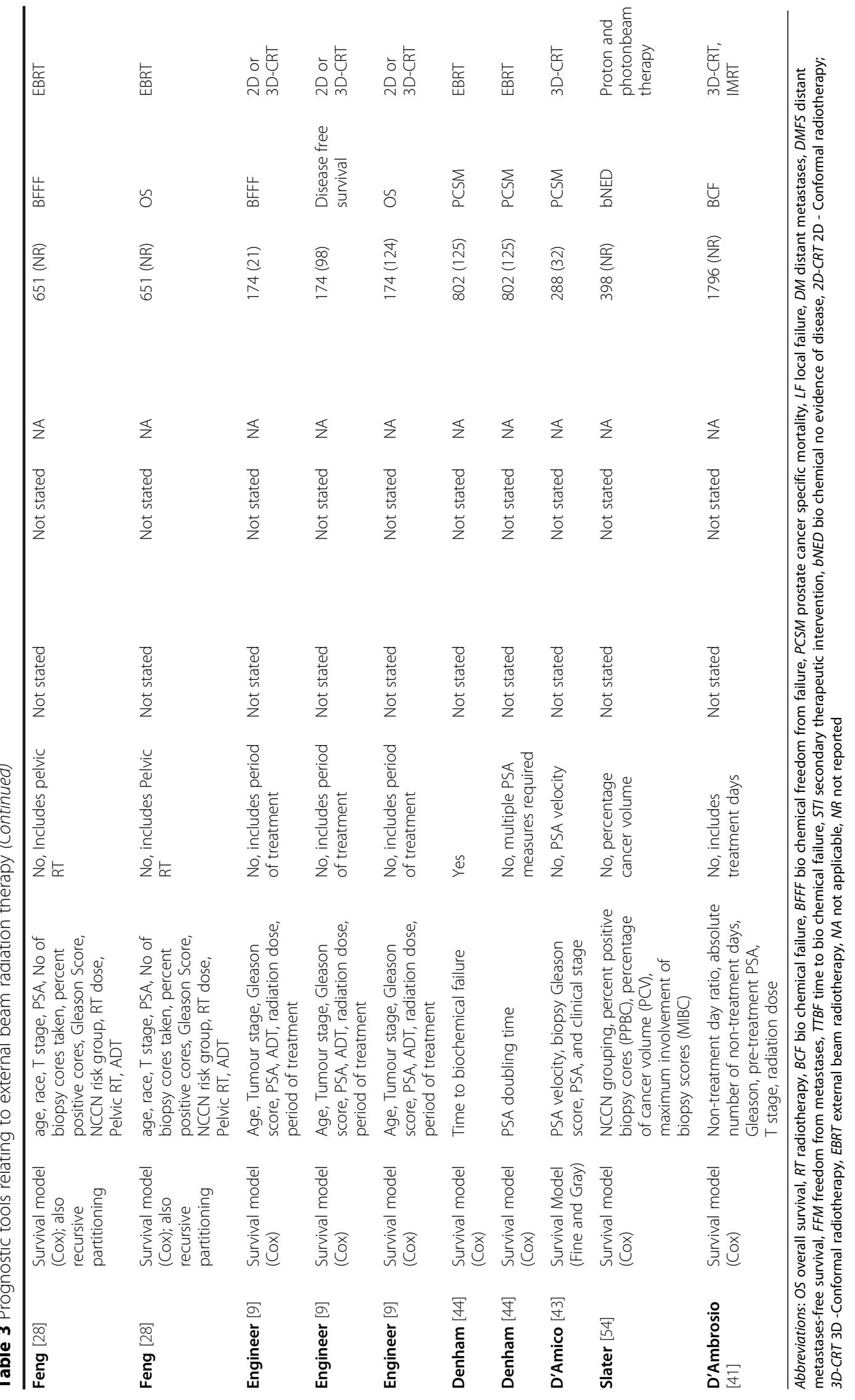




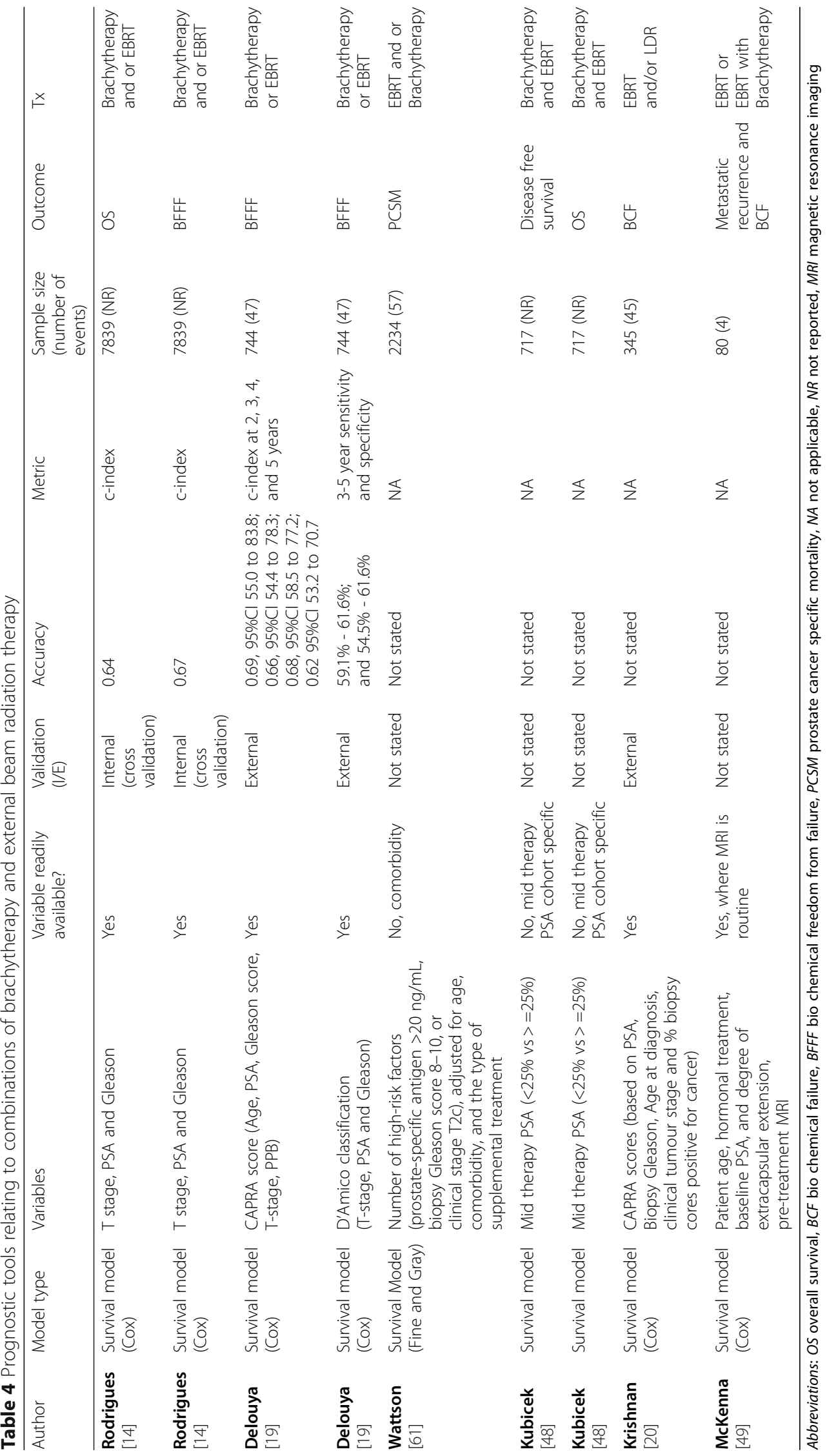


Table $\mathbf{5}$ Risk of bias assessment summary table

\begin{tabular}{|c|c|c|c|c|}
\hline Study Id & Q1 & Q2 & Q3 & Q4 \\
\hline Cooperberg [39] & high & low & low & low \\
\hline Bittner [27] & high & low & high & low \\
\hline Buyyounouski [38] & low & low & low & low \\
\hline Cooperberg (41) & low & high & low & low \\
\hline Delouya [19] & low & high & low & low \\
\hline Engineer [9] & low & high & low & low \\
\hline Feng [28] & low & low & low & low \\
\hline Frank [25] & unclear & high & low & low \\
\hline Frank [45] & unclear & low & unclear & low \\
\hline Halverson [46] & low & low & low & low \\
\hline Huang [47] & low & low & low & low \\
\hline Kaplan [12] & unclear & high & low & low \\
\hline Krishnan [20] & low & high & low & low \\
\hline Kubicek [48] & low & low & low & high \\
\hline Marshall [11] & unclear & low & low & low \\
\hline Potters [16] & unclear & high & low & low \\
\hline Rodrigues [14] & high & unclear & low & low \\
\hline Proust-Lima [51] & low & low & unclear & low \\
\hline Sabolch [53] & low & low & low & low \\
\hline Sanpaolo [21] & low & low & low & low \\
\hline Slater [54] & high & low & low & low \\
\hline Spratt [55] & low & low & low & low \\
\hline Steigler [56] & low & low & low & unclear \\
\hline Taylor [58] & low & low & unclear & low \\
\hline Vainshtein [18] & low & low & low & low \\
\hline Vance [60] & low & low & low & low \\
\hline Wattson [61] & low & high & low & low \\
\hline Westphalen [62] & unclear & high & low & low \\
\hline Williams [17] & low & high & low & low \\
\hline Yoshida [15] & unclear & low & unclear & low \\
\hline Zaorsky [65] & low & low & low & low \\
\hline Zelefsky [10] & low & high & low & low \\
\hline Zelefsky [68] & low & low & low & low \\
\hline Zelefsky [66] & low & low & low & low \\
\hline Zumsteg [69] & low & low & low & low \\
\hline D'Amico [43] & low & high & low & low \\
\hline Yu [64] & low & low & low & low \\
\hline D'Ambrosio [41] & unclear & low & low & low \\
\hline Denham [44] & low & unclear & low & low \\
\hline McKenna [49] & unclear & high & low & high \\
\hline Yu [63] & low & unclear & unclear & low \\
\hline D'Amico [42] & low & low & low & low \\
\hline Zelefsky [67] & low & low & low & low \\
\hline Thames [59] & low & low & unclear & low \\
\hline
\end{tabular}

Table 5 Risk of bias assessment summary table (Continued)

\begin{tabular}{lllll}
\hline Qian [52] & low & low & low & low \\
Sylvester [57] & low & low & low & high \\
Murgic [50] & low & high & low & low \\
Low/47 & $34(72 \%)$ & $30(64 \%)$ & $40(85 \%)$ & $43(91 \%)$ \\
\hline
\end{tabular}

Q1: Was the defined representative sample of patients assembled at a common (usually early) point in the course of their disease)? Q2: Was patient follow-up sufficiently long and complete? Q3: Were outcome criteria either objective or applied in a 'blind' fashion? Q4: If subgroups with different prognoses are identified, did adjustment for important prognostic factors take place? High $=$ high risk of bias, low = low risk of bias, unclear = unclear if study design is at high or low risk of bias

brachytherapy (69\%) did not report model accuracy and among those models which did report accuracy, all related to biochemical failure endpoints. Three studies report to be external validations of the Prostogram nomogram (also known as the Kattan nomogram), all of which have low c-indices $(0.49,0.51$ and 0.66$)$ suggesting that this model is of limited clinical utility. A c-index of 1 indicates a perfect ability to rank the outcomes in the order they actually occurred (100\% sensitivity and specificity), whereas 0.5 is a purely random ranking and is analogous to the area under the receiver operator characteristic curve' (definition from [18]).

The majority of papers identified in this review reported models relating to external beam radiation therapy $(72 / 97=74 \%$ ). Fifty-four percent (39 of 72 ) of these models did not have their accuracy reported. $61 \%$ of models did not report validation (either internal or external, including external validation of already published models).

\section{External beam radiation therapy}

The model relating to external beam radiation therapy with the highest accuracy was described by Vainshtein [19], which was an external validation of the CAPRA stratification in the context of external beam radiation therapy. The cohort included 374 patients and the endpoint of prostate cancer specific mortality was predicted with c-index of 0.86 . Accuracy of this model is also reported for the outcome of biochemical failure and subgroups of patients receiving long term ADT or short term ADT, all which had lower accuracy.

\section{External beam radiation therapy with brachy therapy}

Nine models were identified which were specific to patients treated with external beam radiation therapy in combination with brachytherapy. Of these models, five (56\%) did not report accuracy. The highest accuracy was reported by Delouya [15, 20] (c-index 0.69) predicting biochemical failure free survival at 2-years. This study was based on a cohort of 744 patients and was an external validation of the CAPRA score. Prediction at 5-years was achieved with c-index 0.62 . 


\section{Discussion}

Since the publication of previous reviews, there has been considerable progress in the field of outcomes prediction following prostate cancer treatment. This review identified 47 papers published between 2007 and 2015, which describe 97 predictive tools for men receiving radiotherapy. This includes 66 models which were newly developed and 31 which were validations of already published predictive tools. Consistent with previous reports, most tools $(65 \%)$ are yet to be validated in a population outside the derivation set. Studies were included from 2007 as the modality of radiation therapy has changed significantly over the past decade, and historic data may not be a useful basis for prognosis. Apart from modality, the total dose has also significantly increased however, we found that only five studies [13, 16, 20-22] did not use data from men treated as far back as the 1990s.

The volume of research carried out in the field of prognostics has exploded over the last decade. A systematic review that included all studies published before July 2007 (the cut-off date for inclusion in the present review) identified 17 studies on prognostic models that related to prostate cancer patients treated with radiotherapy [4]. In this review 39 new studies were identified which investigated prognostic markers for BCF. Unfortunately, the majority of new studies did not undertake validation, mirroring the finding of the previous systematic review. As validation - particularly external validation - is vital for the appropriate clinical implementation of prognostic models, this suggests that resources and efforts are not being efficiently targeted to improve tools available for clinical practice.

With regards to the methodological quality of the literature, our critical appraisal found that overall studies were at low to moderate risk of bias. The greatest risk was created by insufficient follow-up (defined as a mean or median of $\geq 5$ years) which only occurred in $64 \%$ of studies. There was also a moderate risk of bias created by the possibility of included patients being at different points in the course of their prostate cancer, however in the majority of cases this was due to insufficient specificity in the description of inclusion criteria as opposed to reported differences. There was little risk of bias created by the measurement of outcomes, as the main outcomes (biochemical failure [various definitions], metastasis, survival) were objective, or by a lack of adjustment for important prognostic factors as the essential factors of prostate cancer prognosis (PSA, Gleason score, and clinical stage) were used nearly universally.

Model accuracy was not reported in $57 \%$ of the models included. Model accuracy was reported to be highest in Vainshtein 2014 [23] with a c-index of 0.86 derived for prediction of prostate cancer specific mortality with the CAPRA score (originally established in [24]), including the addition of variables for the presence of Gleason 5 and treatment with ADT (this c-index relates to patients not receiving ADT). This study acts to externally validate the CAPRA scoring system (with modifications) in patients treated with external beam radiation therapy, though this improvement to the score requires further validation in other populations. Of the remaining 42 models which reported predictive accuracy, c indices were typically in the $0.70-0.80$ range which would be considered 'reasonable' according to Hosmer and Lemeshow [25]. Notably, those papers which did not report external validation typically had higher c-indices suggesting that original model developments should be considered optimistic in their predictive capacity. The lowest c-index $(0.49$, 95\% CI 0.37 to 0.61$)$ was reported for a study [26] performing external validation of the Prostogram nomogram (originally established in [27]) suggesting this nomogram may have little predictive value.

The predictive tools identified in this review included joint-modelling approaches but not neural networks which have featured in previous reviews. This may reflect a change in statistical tools available since publication of earlier catalogues [4]. Two of the survival models $[28,29]$ did not account for competing risks when predicting prostate cancer specific mortality, a potential weakness which could easily be addressed.

The majority of papers attempted prediction relating to biochemical recurrence, prostate cancer specific mortality or overall survival with a smaller subset predicting metastases. Sixteen of the 97 models identified related to brachytherapy with 72 for external beam radiation therapy and 9 a combination of the two. This could reflect more wide-spread use of external beam radiation therapy, and we might anticipate more tools relating to HDR brachytherapy (with or without EBRT) in the future. There is a dearth of externally validated nomograms focusing on brachytherapy and brachytherapy in combination with external beam radiation therapy particularly looking at overall survival and cancer specific survival outcomes.

This study did not explicitly set out to uncover tools incorporating novel variables, but only those which could be used in current clinical settings. Despite this, $31 \%$ of studies included reference to variables which have been less studied to date (e.g. mid-point PSA levels). While such variables may prove useful, there is currently limited opportunity to validate these observations using existing datasets. It is possible that additional variables including standardised measures of comorbidity, imaging features or genetic markers, which are becoming more accessible may help to improve the accuracy of future models. For a recent review of potential molecular and genetic candidate see Hall et al. 2016 [30]. 
Most predictive tools identified in this review were developed in US populations. This observation should be considered by clinicians who are based outside the US when selecting a predictive model to assist treatment decision making. Where possible, tools validated in a setting similar to one's own clinical practice should be selected for use. The number of tools available internationally would be increased with additional validation work conducted outside the US and particularly in multi-national cohorts.

We observed a large degree of variation in the quality of reporting clinical predictive tools. This may stem from the fact that authors are not aware of reporting guidelines in the field or indeed that such guidelines exist. The TRIPOD guidelines (http://www.equator-network.org/reporting-guide lines/tripod-statement/) for reporting of multivariable prediction models were published in March 2015, shortly before the cut-off for papers included in this review. These guidelines have been widely endorsed and published in key journals [31-39]. Further publication of multivariable models would benefit greatly from adherence to these guidelines.

\section{Conclusions}

Tools which aid decision making offer more accurate prediction of clinical outcomes when compared to clinical judgement alone. This understanding has led to a large increase in the number of predictive tools relating to clinical outcomes post radiation therapy between 2007 and 2015. This review identifies 47 papers describing 97 models published in the period, a substantial increase compared to the 17 models previously described between 1966 and 2007. Of the models identified, $65 \%$ had no external validation and $57 \%$ did not report accuracy. Thirty one percent of models included variables which are not part of typical registry data sets, and are therefore difficult to validate. Despite these limitations, there are accurate and externally validated models for external beam radiation therapy treatment which predict prostate cancer specific mortality. There are fewer models which accurately predict outcomes following brachytherapy (alone or in combination with external beam radiation therapy). This review provides an accessible catalogue of predictive tools which could be used currently (i.e. those with high accuracy after external validation) and identifies those which should be prioritised for future validation.

\section{Additional file}

Additional file 1: Reasons for exclusion from the review. (DOCX $21 \mathrm{~kb}$ )

\section{Abbreviations}

BCR: Biochemical recurrence; BF: Biochemical failure; EBRT: External beam radiation therapy; OS: Overall survival; PCSM: Prostate cancer specific mortality; TTP: Time to progression

\section{Acknowledgments}

This project was funded by the Movember Foundation as part of the Prostate Cancer Health Outcomes Research Unit.

Availability of data and materials

All data reported in this publication is publically available.

\section{Authors' contributions}

$\mathrm{ER}$, JC, and MOC conducted the literature searches, screening, appraisal and drafted the manuscript. AV, KB, DR, SE, JM, JM, JZ, MB and KM critically reviewed the manuscript. All authors read and approved the final manuscript.

\section{Competing interests}

The authors declare that they have no competing interests.

\section{Consent for publication}

Not applicable.

Ethics approval and consent to participate

Not applicable.

\section{Author details}

${ }^{1}$ South Australian Prostate Cancer Clinical Outcomes Collaborative (SA-PCCOC), Adelaide, Australia. ${ }^{2}$ Freemasons Foundation Centre for Men's Health, University of Adelaide, Adelaide, Australia. ${ }^{3}$ Centre for Population Health Research, University of South Australia, Adelaide, Australia.

${ }^{4}$ Epidemiology \& Preventative Medicine, Monash University, Clayton, Australia. ${ }^{5}$ Radiation Oncology, Alfred Health, Melbourne, Australia. ${ }^{6}$ Adelaide Radiotherapy Centre, Adelaide, Australia. ${ }^{7}$ SA Health, Repatriation General Hospital, Urology Unit, Daws Road, Daw Park 5041, SA, Australia. ${ }^{8}$ Flinders Centre for Innovation in Cancer, Bedford Park, Australia. ${ }^{9}$ Joanna Briggs Institute, University of Adelaide, Adelaide, Australia. ${ }^{10}$ Discipline of Surgery, University of Adelaide, Adelaide, Australia. ${ }^{11}$ School of Public Health and

Preventive Medicine, Monash University, Clayton, Australia.

Received: 1 August 2016 Accepted: 22 February 2017

Published online: 21 March 2017

\section{References}

1. Global Burden of Disease Cancer C, Fitzmaurice C, Dicker D, et al. The global burden of cancer 2013. JAMA. 2015;1:505-27.

2. Heidenreich A, Bellmunt J, Bolla M, et al. EAU guidelines on prostate cancer. Part 1: screening, diagnosis, and treatment of clinically localised disease. Eur Urol. 2011;59:61-71.

3. Mottet N, Bellmunt J, Bolla M, et al. EAU guidelines on prostate cancer. Part II: treatment of advanced, relapsing, and castration-resistant prostate cancer. Eur Urol. 2011;59:572-83.

4. Shariat SF, Karakiewicz PI, Roehrborn CG, Kattan MW. An updated catalog of prostate cancer predictive tools. Cancer. 2008;113:3075-99.

5. Ross PL, Gerigk C, Gonen M, et al. Comparisons of nomograms and urologists' predictions in prostate cancer. Semin Urol Oncol. 2002;20:82-8.

6. Walz J, Gallina A, Perrotte P, et al. Clinicians are poor raters of lifeexpectancy before radical prostatectomy or definitive radiotherapy for localized prostate cancer. BJU Int. 2007:100:1254-8.

7. Cohen RJ, O'Brien BA. Comparative analysis of three risk assessment tools in Australian patients with prostate cancer. BJU Int. 2011;108 Suppl 2:56-7.

8. Evans SM, Nag N, Roder D, et al. Development of an international prostate cancer outcomes registry. BJU Int. 2016;117 Suppl 4:60-7.

9. Medicine CfE-B. Critical appraisal of Prognostic studies. 2016 [cited 2016]; Available from: http://www.cebm.net/critical-appraisal/

10. Engineer R, Bhutani R, Mahantshetty U, Murthy V, Shrivastava SK. From twodimensional to three-dimensional conformal radiotherapy in prostate cancer: an Indian experience. Indian J Cancer. 2010;47:332-8.

11. Zelefsky MJ, Chou JF, Pei X, et al. Predicting biochemical tumor control after brachytherapy for clinically localized prostate cancer: The Memorial SloanKettering Cancer Center experience. Brachytherapy. 2012;11:245-9.

12. Marshall RA, Buckstein M, Stone NN, Stock R. Treatment outcomes and morbidity following definitive brachytherapy with or without external beam radiation for the treatment of localized prostate cancer: 20-year experience at Mount Sinai Medical Center. Urol Oncol. 2014;32:38. e1-7. 
13. Kaplan A, German L, Chen J, Matzkin H, Mabjeesh NJ. Validation and comparison of the two Kattan nomograms in patients with prostate cancer treated with 125iodine brachytherapy. BJU Int. 2012;109:1661-5.

14. McKenna DA, Coakley FV, Westphalen AC, et al. Prostate cancer: role of pretreatment MR in predicting outcome after external-beam radiation therapy-initial experience. Radiology. 2008;247(1):141-6.

15. Rodrigues $G$, Lukka $H$, Warde $P$, et al. The prostate cancer risk stratification (ProCaRS) project: recursive partitioning risk stratification analysis. Radiother Oncol. 2013;109:204-10.

16. Yoshida K, Yamazaki H, Nakamura S, et al. Role of novel risk classification method, Prostate Cancer Risk Index (PRIX) for clinically localized prostate cancer after high-dose-rate interstitial brachytherapy as monotherapy. Anticancer Res. 2014:34:3077-81.

17. Potters L, Roach IM, Davis BJ, et al. Postoperative nomogram predicting the 9-year probability of prostate cancer recurrence after permanent prostate brachytherapy using radiation dose as a prognostic variable. Int J Radiat Oncol Biol Phys. 2010;76:1061-5.

18. Williams SG, Buyyounouski MK, Pickles T, et al. Percentage of biopsy cores positive for malignancy and biochemical failure following prostate cancer radiotherapy in 3,264 Men: statistical significance without predictive performance. Int J Radiat Oncol Biol Phys. 2008;70:1169-75.

19. Vainshtein JM, Schipper M, Vance S, Feng FY, Olson KB, Hamstra DA. Limitations of the cancer of the prostate risk assessment (CAPRA) prognostic tool for prediction of metastases and prostate cancer-specific mortality in patients treated with external beam radiation therapy. Cancer Clinical Trials: American Journal of Clinical Oncology; 2014.

20. Delouya G, Krishnan V, Bahary JP, Larrivee S, Taussky D. Analysis of the cancer of the prostate risk assessment to predict for biochemical failure after external beam radiotherapy or prostate seed brachytherapy. Urology. 2014;84:629-33.

21. Krishnan V, Delouya G, Bahary JP, Larrivee S, Taussky D. The Cancer of the Prostate Risk Assessment (CAPRA) score predicts biochemical recurrence in intermediate-risk prostate cancer treated with external beam radiotherapy (EBRT) dose escalation or low-dose rate (LDR) brachytherapy. BJU Int. 2014;114:865-71.

22. Sanpaolo P, Barbieri V, Genovesi D. Biologically effective dose and definitive radiation treatment for localized prostate cancer. Treatment gaps do affect the risk of biochemical failure. Strahlenther Onkol. 2014;190:732-8.

23. Vainshtein JM, Schipper M, Vance S, Feng FY, Olson KB, Hamstra DA. Limitations of the Cancer of the Prostate Risk Assessment (CAPRA) Prognostic Tool for Prediction of Metastases and Prostate Cancer-specific Mortality in Patients Treated With External Beam Radiation Therapy. Am J Clin Oncol. 2014

24. Cooperberg MR, Pasta DJ, Elkin EP, et al. The University of California, San Francisco Cancer of the Prostate Risk Assessment score: a straightforward and reliable preoperative predictor of disease recurrence after radical prostatectomy. J Urol. 2005;173:1938-42.

25. S. HDWaL. Applied logistic regression. 2nd ed. New York: Wiley; 2000.

26. Frank SJ, Levy LB, Kuban DA, et al. Prostogram predicted brachytherapy outcomes are Not universally accurate: an analysis based on the M. D. Anderson cancer center experience with 125lodine brachytherapy. J Urol. 2009;181:1658-64.

27. Kattan MW, Potters L, Blasko JC, et al. Pretreatment nomogram for predicting freedom from recurrence after permanent prostate brachytherapy in prostate cancer. Urology. 2001;58:393-9.

28. Bittner N, Merrick GS, Galbreath RW, Butler WM, Adamovich E, Wallner KE. Greater biopsy core number is associated with improved biochemical control in patients treated with permanent prostate brachytherapy. Int J Radiat Oncol Biol Phys. 2010;78:1104-10.

29. Feng FY, Qian $Y$, Stenmark MH, et al. Perineural invasion predicts increased recurrence, metastasis, and death from prostate cancer following treatment with dose-escalated radiation therapy. Int J Radiat Oncol Biol Phys. 2011;81:e361-7.

30. Hall WA, Lawton CA, Jani AB, Pollack A, Feng FY. Biomarkers of outcome in patients with localized prostate cancer treated with radiotherapy. Seminars in radiation oncology: Elsevier; 2017. p. 11-20.

31. Collins GS, Reitsma JB, Altman DG, Moons KG. Transparent reporting of a multivariable prediction model for individual prognosis or diagnosis (TRIPOD): the TRIPOD statement. Ann Intern Med. 2015;162:55-63.

32. Moons KG, Altman DG, Reitsma JB, et al. Transparent Reporting of a multivariable prediction model for Individual Prognosis or Diagnosis (TRIPOD): explanation and elaboration. Ann Intern Med. 2015;162:W1-73.
33. Collins GS, Reitsma JB, Altman DG, Moons KG. Transparent reporting of a multivariable prediction model for individual prognosis or diagnosis (TRIPOD): the TRIPOD statement. Br J Cancer. 2015;112:251-9.

34. Collins GS, Reitsma JB, Altman DG, Moons KG. Transparent reporting of a multivariable prediction model for individual prognosis or diagnosis (TRIPOD): the TRIPOD Statement. BMC Med. 2015;13:1.

35. Collins GS, Reitsma JB, Altman DG, Moons KG. Transparent reporting of a multivariable prediction model for individual prognosis or diagnosis (TRIPOD): the TRIPOD statement. BMJ. 2015;350:97594.

36. Collins GS, Reitsma JB, Altman DG, Moons KG. members of Tg. Transparent Reporting of a Multivariable Prediction Model for Individual Prognosis or Diagnosis (TRIPOD): The TRIPOD Statement. Eur Urol. 2015;67:1142-51.

37. Collins GS, Reitsma JB, Altman DG, Moons KG. Transparent Reporting of a multivariable prediction model for Individual Prognosis Or Diagnosis (TRIPOD): the TRIPOD Statement. Br J Surg. 2015;102:148-58.

38. Collins GS, Reitsma JB, Altman DG, Moons KG. Transparent Reporting of a multivariable prediction model for Individual Prognosis Or Diagnosis (TRIPOD). Ann Intern Med. 2015;162:735-6.

39. Moons KG, Altman DG, Reitsma JB, Collins GS. New guideline for the reporting of studies developing, validating, or updating a multivariable clinical prediction model: the TRIPOD statement. Adv Anat Pathol. 2015;22:303-5.

40. Cooperberg MR, Vickers AJ, Broering JM, Carroll PR. Comparative riskadjusted mortality outcomes after primary surgery, radiotherapy, or androgen-deprivation therapy for localized prostate cancer. Cancer. 2010; 116(22):5226-34. Pubmed Central PMCID: PMC2975879, Epub 2010/08/07. eng

41. D'Ambrosio DJ, Li T, Horwitz EM, Chen DY, Pollack A, Buyyounouski MK. Does treatment duration affect outcome after radiotherapy for prostate cancer? Int J Radiat Oncol Biol Phys. 2008;72(5):1402-7. Pubmed Central PMCID: Pmc2763099, Epub 2008/05/13. eng.

42. D'Amico AV, Moran BJ, Braccioforte MH, Dosoretz D, Salenius S, Katin M, et al. Risk of death from prostate cancer after brachytherapy alone or with radiation, androgen suppression therapy, or both in men with high-risk disease. J Clin Oncol. 2009;27(24):3923-8. Epub 2009/07/15. eng.

43. D'Amico AV, Chen MH, Catalona WJ, Sun L, Roehl KA, Moul JW. Prostate cancer-specific mortality after radical prostatectomy or external beam radiation therapy in men with 1 or more high-risk factors. Cancer. 2007;110(1):56-61.

44. Denham JW, Steigler A, Wilcox C, Lamb DS, Joseph D, Atkinson C, et al. Time to biochemical failure and prostate-specific antigen doubling time as surrogates for prostate cancer-specific mortality: evidence from the TROG 96.01 randomised controlled trial. Lancet Oncol. 2008;9(11):1058-68.

45. Frank SJ, Levy LB, van Vulpen M, Crook J, Sylvester J, Grimm P, et al Outcomes after prostate brachytherapy are even better than predicted. Cancer. 2012;118(3):839-47. Epub 2011/07/14. eng.

46. Halverson S, Schipper M, Blas K, Lee V, Sabolch A, Olson K, et al. The Cancer of the Prostate Risk Assessment (CAPRA) in patients treated with external beam radiation therapy: Evaluation and optimization in patients at higher risk of relapse. Radiother Oncol. 2011;101(3):513-20.

47. Huang J, Vicini FA, Williams SG, Ye H, McGrath S, Ghilezan M, et al. Percentage of positive biopsy cores: a better risk stratification model for prostate cancer? Int J Radiat Oncol Biol Phys. 2012;83(4):1141-8. Epub 2011/11/22. eng.

48. Kubicek GJ, Naguib M, Redfield S, Grayback N, Olszanski A, Dawson G, et al. PSA decrease during combined-modality radiotherapy predicts for treatment outcome. Int J Radiat Oncol Biol Phys. 2010;78(3):759-62.

49. McKenna DA, Coakley FV, Westphalen AC, Zhao S, Lu Y, Webb EM, et al. Prostate cancer: role of pretreatment MR in predicting outcome after external-beam radiation therapy-initial experience. Radiology. 2008; 247(1):141-6. Pubmed Central PMCID: 3694427.

50. Murgic J, Stenmark MH, Halverson S, Blas K, Feng FY, Hamstra DA. The role of the maximum involvement of biopsy core in predicting outcome for patients treated with dose-escalated radiation therapy for prostate cancer. Radiation oncology (London, England). 2012;7:127. Pubmed Central PMCID: 3484035.

51. Proust-Lima C, Taylor JM. Development and validation of a dynamic prognostic tool for prostate cancer recurrence using repeated measures of posttreatment PSA: a joint modeling approach. Biostatistics (Oxford, England). 2009;10(3):535-49. Pubmed Central PMCID: PMC2697347. Epub 2009/04/17. eng.

52. Qian Y, Feng FY, Halverson S, Blas K, Sandler HM, Hamstra DA. The percent of positive biopsy cores improves prediction of prostate cancer-specific death in patients treated with dose-escalated radiotherapy. Int J Radiat Oncol Biol Phys. 2011;81(3):e135-42. 
53. Sabolch A, Feng FY, Daignault-Newton S, Halverson S, Blas K, Phelps L, et al. Gleason Pattern 5 is the greatest risk factor for clinical failure and death from prostate cancer after dose-escalated radiation therapy and hormonal ablation. Int J Radiat Oncol Biol Phys. 2011;81(4):e351-60.

54. Slater JM, Bush DA, Grove R, Slater JD. The prognostic value of percentage of positive biopsy cores, percentage of cancer volume, and maximum involvement of biopsy cores in prostate cancer patients receiving proton and photon beam therapy. Technol Cancer Res Treat. 2014;13(3):227-31.

55. Spratt DE, Pei X, Yamada J, Kollmeier MA, Cox B, Zelefsky MJ. Long-term survival and toxicity in patients treated with high-dose intensity modulated radiation therapy for localized prostate cancer. Int J Radiat Oncol Biol Phys. 2013;85(3):686-92.

56. Steigler A, Denham JW, Lamb DS, Spry NA, Joseph D, Matthews J, et al. Risk stratification after biochemical failure following curative treatment of locally advanced prostate cancer: Data from the TROG 96.01 trial. Prostate cancer. 2012.

57. Sylvester JE, Grimm PD, Wong J, Galbreath RW, Merrick G, Blasko JC. Fifteen-year biochemical relapse-free survival, cause-specific survival, and overall survival following I(125) prostate brachytherapy in clinically localized prostate cancer: Seattle experience. Int J Radiat Oncol Biol Phys. 2011; 81(2):376-81.

58. Taylor JM, Park Y, Ankerst DP, Proust-Lima C, Williams S, Kestin L, et al. Realtime individual predictions of prostate cancer recurrence using joint models. Biometrics. 2013;69(1):206-13. Pubmed Central PMCID: Pmc3622120, Epub 2013/02/06. eng.

59. Thames HD, Kuban D, Levy LB, Horwitz EM, Kupelian P, Martinez A, et al. The role of overall treatment time in the outcome of radiotherapy of prostate cancer: an analysis of biochemical failure in 4839 men treated between 1987 and 1995. Radiother Oncol. 2010;96(1):6-12. Epub 2010/04/20. eng.

60. Vance SM, Stenmark MH, Blas K, Halverson S, Hamstra DA, Feng FY. Percentage of cancer volume in biopsy cores is prognostic for prostate cancer death and overall survival in patients treated with doseescalated external beam radiotherapy. Int J Radiat Oncol Biol Phys. 2012;83(3):940-6. Epub 2011/11/08. eng.

61. Wattson DA, Chen MH, Moul JW, Moran BJ, Dosoretz DE, Robertson CN, et al. The number of high-risk factors and the risk of prostate cancer-specific mortality after brachytherapy: Implications for treatment selection. Int J Radiat Oncol Biol Phys. 2012;82(5):e773-9.

62. Westphalen AC, Koff WJ, Coakley FV, Muglia VF, Neuhaus JM, Marcus RT, et al. Prostate cancer: Prediction of biochemical failure after external-beam radiation therapy - Kattan nomogram and endorectal MR imaging estimation of tumor volume. Radiology. 2011;261(2):477-86. English.

63. Yu M, Taylor JMG, Sandler HM. Individual Prediction in Prostate Cancer Studies using a Joint Longitudinal Survival-Cure Model. J Am Stat Assoc. 2008;103(481):178-87.

64. Yu H-HM, Song DY, Tsai Y-Y, Thompson T, Frassica DA, DeWeese TL. Perineural invasion affects biochemical recurrence-free survival in patients with prostate cancer treated with definitive external beam radiotherapy. Urology. 2007;70(1):111-6.

65. Zaorsky NG, Li T, Devarajan K, Horwitz EM, Buyyounouski MK. Assessment of the American Joint Committee on Cancer staging (sixth and seventh editions) for clinically localized prostate cancer treated with external beam radiotherapy and comparison with the National Comprehensive Cancer Network riskstratification method. Cancer. 2012;118(22):5535-43. Pubmed Central PMCID: PMC3410044, Epub 2012/05/01. eng.

66. Zelefsky MJ, Pei X, Chou JF, Schechter M, Kollmeier M, Cox B, et al. Dose escalation for prostate cancer radiotherapy: Predictors of long-term biochemical tumor control and distant metastases-free survival outcomes. Eur Urol. 2011;60(6):1133-9. English.

67. Zelefsky MJ, Shi W, Yamada Y, Kollmeier MA, Cox B, Park J, et al. Postradiotherapy 2-year prostatespecific antigen nadir as a predictor of long-term prostate cancer mortality. Int J Radiat Oncol Biol Phys. 2009;75(5):1350-6. Epub 2009/06/12. eng.

68. Zelefsky MJ, Kattan MW, Fearn P, Fearon BL, Stasi JP, Shippy AM, et al. Pretreatment nomogram predicting ten-year biochemical outcome of three-dimensional conformal radiotherapy and intensitymodulated radiotherapy for prostate cancer. Urology. 2007;70(2):283-7.

69. Zumsteg ZS, Spratt DE, Pei I, Zhang Z, Yamada Y, Kollmeier M, et al. A new risk classification system for therapeutic decision making with intermediate-risk prostate cancer patients undergoing dose-escalated external-beam radiation therapy. Eur Urol. 2013;64(6):895-902

\section{Submit your next manuscript to BioMed Central and we will help you at every step:}

- We accept pre-submission inquiries

- Our selector tool helps you to find the most relevant journal

- We provide round the clock customer support

- Convenient online submission

- Thorough peer review

- Inclusion in PubMed and all major indexing services

- Maximum visibility for your research

Submit your manuscript at www.biomedcentral.com/submit
Biomed Central 